\title{
Spectral reproduction: drivers, use cases and workflow
}

\author{
Tanzima Habib, Phil Green and Peter Nussbaum, Norwegian University of Science and Technology, Gjøvik, Norway
}

\begin{abstract}
This paper identifies the drivers that enable spectral reproduction today and have the ability to increase its usage in the future. Different use cases of spectral reproduction in the field of colour imaging are discussed along with some of the possible workflows. Some workflows are further divided into input, spectral processing and output units as an example, such that the individual spectral processing units that can be encoded in an ICC profile can be identified. It also gives a map of how some common processing elements can be connected in order to constitute a spectral reproduction system.
\end{abstract}

\section{Introduction}

The majority of today's colour reproduction systems are based on trichromacy, whereby the three cones of the human visual system are stimulated in ratios that match those of the desired appearance. Trichromatic reproduction is efficient, requiring only three primaries, and there can be considerable flexibility in the choice of primaries for particular reproduction goals. Nevertheless, trichromatic reproduction has a number of limitations, the most obvious being that matches are metameric and may not hold when the spectral power distribution of the viewing illumination varies.

Spectral data are being increasingly used at all stages of the colour reproduction workflow. This demand has also led to the evolution of spectral acquisition systems that can capture narrow band spectral data with precision, processors that can compute and handle data faster, storage devices that are portable and can store large amount data and output devices that has wider/bigger gamut to reproduce a close match to the original colour. With the availability of multispectral and hyperspectral cameras and other spectral imaging devices, spectral and multi-channel data is now more abundant. This has enabled the acquisition of surface reflectance that holds accurate colour information of a scene. For example, using reflectance samples, spectral characterisation of printers is achieved where the device dependent space is described by a reflectance function. In the colorant industries, spectral reflectance data of inks and paints help minimise metameric effects. In cultural heritage conservation, spectral data is used to characterise materials and used for restoration.

It may also be necessary to transform from a colorimetric domain to a higher dimension spectral domain for intermediate processing. For example, watermarking images in the spectral domain can make the watermark imperceptible [1]. It also enables the use of metameric samples to hide data for a range of illuminants. Spectral reproduction can also be used to perform chromatic adaptation [2]. An intermediate spectral reproduction step can help create an image that looks like a real scene using a photorealistic rendering system.

Because spectral data provides more information about material properties and enables increase accuracy for colour matching, spectrally-defined colour reproduction is in demand in fields like medical imaging, cultural heritage, (artwork reproduction, textiles etc. Graphic communication industries are especially interested in the use of spectral data to create accurate reproduction of spot colours. Spectral data can be used to reduce metameric effects, which is important for colour critical work and for accurate characterisation of devices [3] [4].

Many spectral reconstruction methods have been developed to enable conversion between three-component data and spectral data. Simple methods like principal component analysis [5] [6] or pseudo inverse methods [7]are frequently used, while more complex methods use neural network, support vector regression and constrained linear programming [8]. Below we review an example of such methods within a spectral reproduction workflow and the current colour management architecture.

A complete spectrally based colour management framework is now possible where the input, the output and all intermediate processing can be spectral, by making use of the iccMAX colour management architecture. It is also anticipated that a colour appearance framework can be better defined using spectral information where BRDF data can be used to model gloss and texture in addition to colour.

Although technology today provides a strong basis for the implementation of spectral reproduction workflows, there are still certain physical limitations [9]:

-original scene spectra cannot have a complete match with the spectra reproduced by the output device or there cannot be a complete match between reproductions by different media devices (example a display and a print)

- there is usually a difference in the dynamic range of the original scene and the output device

We address these issues by mapping the original scene to the reproduction gamut that best represents the appearance of the original. (The evaluation of the quality of reproduction is outside the scope of this paper.) In this paper, we review spectral acquisition and processing, discuss the different use cases of spectral reproduction that are being used today and identify some of the spectral reproduction workflows and methods of connecting their different components.

\section{Spectral acquisition and processing}

Spectral acquisition is the capturing of image data over wavelength intervals smaller than be found in broadband trichromatic capture. Multispectral and hyperspectral imaging can also be based on wavelength intervals outside the visible spectrum (although here we consider only the visible spectrum). Measurement instruments can capture surface reflectance or transmittance of an object, or the spectral radiance or irradiance of a luminous source. Multispectral and hyperspectral imaging devices are increasingly used in applications such as medical imaging and cultural heritage, where precise spectral data of a scene is critical for image capture 
and possibly reproduction [9]. Spectral processing methods such as spectral estimation can be applied to multispectral or colorimetric data with a priori knowledge to convert them to spectral reflectance or radiance when required instead of using hyperspectral data that requires larger storage or bandwidth to transmit over the internet [10].

Various spectral processing steps are required to achieve a reproduction aim. Noise removal, dimensionality increase or decrease, transformations from spectral to colorimetry, spectral separation, spectral matching, gamut mapping etc. can be performed. These processes can be computationally expensive and hence the encoding of spectral reproduction workflows within colour management should be carefully designed for computational efficiency. We will discuss a few examples in the following sections.

\section{Spectral Reproduction} below:

Some of the use cases of spectral reproduction are discussed

\section{Use case 1: Spot colour reproduction}

In printing, it is often required to match a spot colour using process colours for example to reproduce a brand colour on a document. In such a case, the spectral reflectance of the spot colour is used to find the best spectral match that can be obtained with the process colours. The reflectance of the spot colour is stored in the ICC profile and/or PDF file using the $\mathrm{CxF} / \mathrm{x} 4$ file format [11], and a spectral matching algorithm can then be used to find the best combination of process colours. Similar spectral characterisation can be done for the reflectances of spot colour tints and overprints. Another example for spectral reproduction is the prediction of the colorimetry of spot colour overprints of two inks for the purpose of previewing using a linear combination of the reflectances of the two inks $[12,13]$.

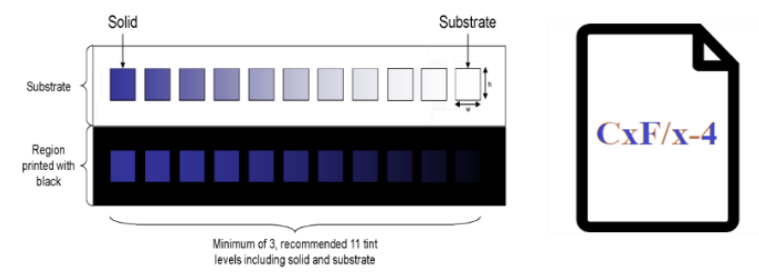

Figure 1. Spot colour reproduction

\section{Use case 2: Ink switching}

When an ink is switched or an ink sequence is changed as shown in figure 2., a printer needs to be characterised to map the new state. This can be done using spectral characterisation and $\mathrm{CxF}$ 4 files to access the reflectance data. Many different spectral characterisation algorithms exist [3] [14] [15].

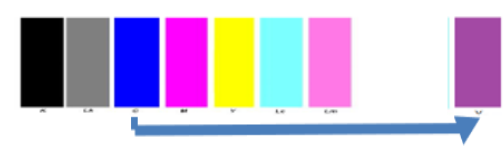

Figure 2. Ink switching

\section{Use case 3: Substrate colour adjustment}

Substrate correction adjusts colorimetry to compensate for substrate colour in order to obtain a visual match. A degree of adaptation is applied, depending on how close the substrate white point is to the reference white point. data method for achieving this colorimetrically is defined in ISO 12647-2. If this adjustment is computed in the spectral domain, a new way to apply partial adaptation to the spectral reproduction can be developed instead of only performing media relative adjustment. This use case is for future consideration.

\section{Use case 4: Data hiding}

Watermarking objects for copyright protection, temper detection, authentication etc. is another use case where spectral data of an image can be used for such embedding. Metameric pairs of a colorant can be used to implement the watermarks. A few methods were suggested by Bala et. al [1]. Metameric colours can be used to create an image and to embed corresponding watermarks that can be easily deciphered by an infrared light/or narrow band illumination/ or using UV light (figure 3) depending on the characteristics of the watermark [1]. Spectral estimation of colorimetry before the watermarking stage is essential in this workflow.

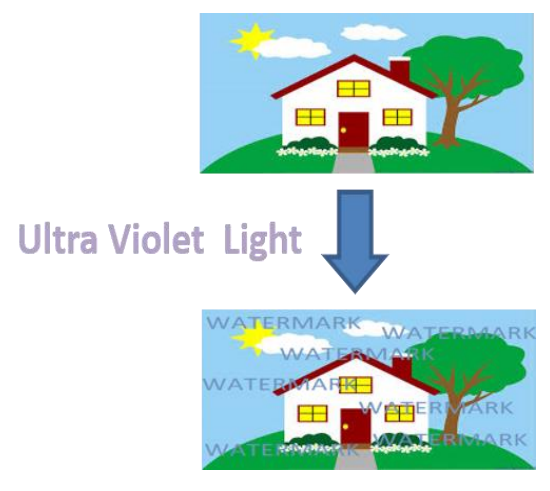

Figure 3. Watermarking using metameric colours [1]

\section{Use case 5: Scene referred spectral reproduction}

In order to create an image that is similar to that perceived by an observer looking at a real scene, a photorealistic rendering system is used [16] [17]. In this case a database of natural spectra is required that would match the visual impression of an observer to virtual scenes. Apart from that, different physical effects such as fluorescence, diffraction, polarization etc. must be supported by the rendering system which is not possible using a tristimulus transport which is dependent on the colour space. It has been pointed out [18], that spectral space has to be used in a physically meaningful rendering system where the output is to be used for predictive purposes. With the advent of High Dynamic Range and Wide Colour Gamut devices, the photorealistic rendered images can be accurately handled. Also, the new colour management architecture can be used to create profiles for such realistic and spectral based rendering.

\section{Use case 6: Hi-fidelity colour reproduction}

Hi-fidelity colour reproduction is important in medical imaging and reproduction of art works. In the case of medical imaging, hifidelity colour as well as spectral data can help quantify diseases and assess treatments [19] [10]. Spectral data of a scene can be used to reproduce a scene that is colorimetrically accurate. When spectral 
images are compressed then one has to ensure that the reconstruction process enables both colorimetric accuracy and spectral accuracy. This ensures that the hi-fidelity of colour reproduction will be preserved under different illumination.

\section{Use case 7: Spectral BRDF}

Bi-directional reflectance distribution function (BRDF) is used to render synthetic images at different orientations of the viewing angle and the illumination angles. This is important for reproducing the colour of an object in images as the direction of light or viewing changes. This can be achieved by computing the new reflectance per pixel based on parameters obtained from a BRDF model such as Ward and Cook-Torrance. There are many ways to implement BRDF using iccMAX. BRDF tags can be used to obtain BRDF parameters using an ICC profile or a BRDF model can be encoded with its optimized parameters in an ICC profile to render reflectances directly per pixel by applying the profile. For optimization of parameters, a database of BRDF measurements are required. Many image-based BRDF measurement set ups have been proposed over the years for faster acquisition [20] [21] [22] [23] [24]. Once such set up has been proposed by Sole et. al [25] where the sample on a circular frame is fixed and shot by $\mathrm{RGB} /$ multispectral camera for different camera(viewing) and light source angles. A BRDF rendering workflow is given in figure 4 .

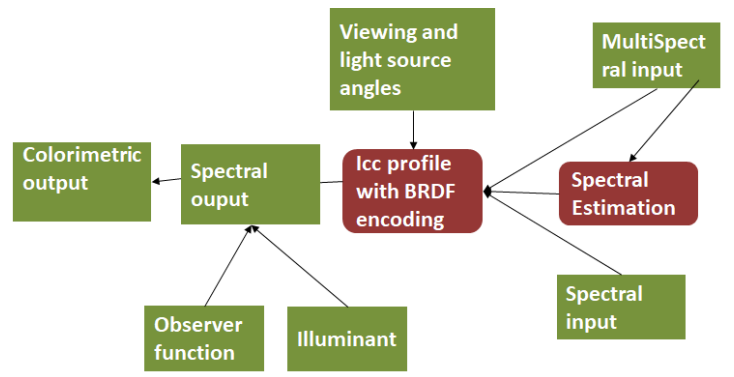

Figure 4. A spectral BRDF Workflow

\section{Use case 8: Reducing metameric mismatch}

In colour reproduction, it is desirable that the prints from two different printing devices matched under different illuminants. For this the two prints have to be matched spectrally. While an exact spectral match is difficult, the best scenario is to minimize the degree of metameric mismatch for prints or printed objects for a set of illuminants [26] [27]. This is especially important for packaging industries, fine arts, catalogues etc. One such workflow is proposed by Coppel et. al. [27] where spectral separation is carried out by choosing the ink combinations that has spectral match to the target spectra such that that they are within a chosen colour difference threshold under D50 illuminant, from these chosen set again the final ink combination is chosen such that it produces the least colour difference under different set of illuminants. This particular method was inspired by a spectral gamut mapping method described by Urban and Roy [28]. A generic spectral reproduction workflow is given in figure 5 .

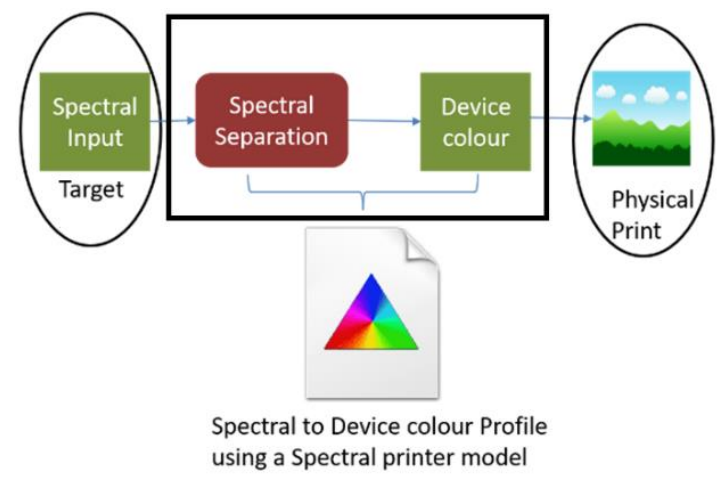

Figure 5. A spectral proofing workflow

\section{Use case 9: Display matching with custom observers}

Differences in colour matching functions between observers leads to problems in matching colorimetry on a display. This is called observer metamerism [29]. Wide Colour Gamut displays have spectrally narrow primaries which increases the degree of observer metamerism. One approach is to apply individual colour matching functions to spectra to obtain a personalized reproduction. There are many studies that discuss observer metamerism and the need for personalized softproofing [30] [31] [32] [33]. One such workflow can be when we wish to match Observer 1's print to Observer 2's colour perception, where we can use the colour matching function of Observer 1 to characterise a printer and apply this profile to reproduce a print that matches the colour perception of observer 2 .

\section{Connecting spectral reproduction workflows}

All the above spectral reproduction use cases have some common components in their workflows. The generic workflow is, given an input image (colorimetric, spectral or multispectral) we perform some intermediate spectral processing and output an image that satisfies a reproduction goal. Processing units such as BRDF processing, process colour matching, spectral characterisation, and custom observer colour matching represent the reproduction goals while processing modules such as spectral estimation, spectral gamut mapping etc. are the common intermediate processing units that can be used by any workflow. The input to such a spectral reproduction system can be colorimetric, spectral or multi-spectral data with the flexibility to pass more parameters (e.g. viewing angle, illumination angle) as input while the output can be obtained in colorimetric, spectral or multispectral domain. Figure 6 gives an overview of such a spectral reproduction system whose individual components can be envisioned to be encoded and connected using the new colour management architecture. This new colour management architecture is called iccMAX and it is an extension of ICC V4, developed to cater to requirements such as support for spectral data, flexibility in using any illuminant or colour matching function, programmable transforms using calculator element etc [34] [35].

Encoding a spectral estimation method using iccMAX has been successfully implemented in which a profile is created to produce 
the estimated reflectance of an XYZ image. An intermediate transformation step is included in the profile to convert images from other colour spaces to XYZ before the spectral estimation process. This image can then be used for further processing to achieve a reproduction aim. A good example of BRDF implementation using iccMAX is described by Vogh [36].

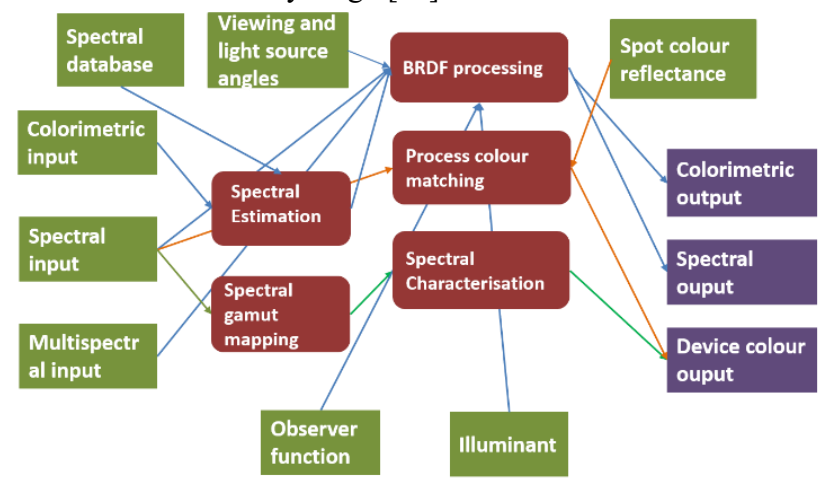

Figure 6. Connecting different spectral reproduction units

\section{Conclusion}

This paper reviews spectral reproduction use cases and workflows to establish the necessity of moving towards a spectral colour management. Spectral estimation, BRDF rendering etc. using spectral ICC profile is now possible. These researches can open new doors towards achieving other spectral reproduction goals. But certain needs have yet to be addressed to create an efficient and consistent spectral reproduction system. Some of these requirements are - standardization of intermediate spectral processing units such as spectral estimation, more object specific spectral database has to be created, new metrics to assess the quality of spectral reproduction are required and there should be support for multichannel profiles in existing graphic editors.

\section{Author Biography}

Tanzima Habib is a PhD scholar at NTNU, Norway. She has a diverse background in software engineering and geoinformatics, and her interest lies primarily in the domain of colour management and image processing.

Phil Green is Professor of Colour Imaging at the Colour and Visual Computing Laboratory, NTNU, Norway. He is also Technical Secretary of the International Colour Consortium.

Peter Nussbaum is Associate Professor at NTNU, Gjøvik within the Faculty of Computer Science and Media Technology and Department of Computer Science.

\section{References}

[1] R. Bala, K. M. Braun and R. P. Loce, "Watermark encoding and detection using narrowband illumination," in Color and Imaging Conference, 2009.

[2] S. A. Burns, "Chromatic adaptation transform by spectral reconstruction," Color Research \& Application., 2019.

[3] Y. Chen, R. S. Berns and L. A. Taplin, "Six color printer characterization using an optimized cellular Yule-Nielsen spectral
Neugebauer model," Journal of Imaging Science and Technology, vol. 48 , no. 6 , pp. 519-528, 2004.

[4] M.-C. Lo and C.-L. ,. H. T.-H. Chen, "Characterization of HighFidelity Color Printing Devices Using Illuminant-Independent Approaches for Color Imaging Application.," NIP \& Digital Fabrication Conference, vol. 2, pp. 597-602, 2008.

[5] H. S. Fairman and M. H. Brill, "The principal components of reflectances," Color Research \& Application: Endorsed by InterSociety Color Council, The Colour Group (Great Britain), Canadian Society for Color, Color Science Association of Japan, Dutch Society for the Study of Color, The Swedish Colour Centre Foundation, Colour Soc, vol. 29, no. 2, pp. 104-110, 2004.

[6] F. Agahian, S. A. Amirshahi and S. H. Amirshahi, "Reconstruction of reflectance spectra using weighted principal component analysis," Color Research \& Application: Endorsed by Inter-Society Color Council, The Colour Group (Great Britain), Canadian Society for Color, Color Science Association of Japan, Dutch Society for the Study of Color, The Swedish Colour Centre Foundation, Colour Soc, vol. 33, no. 5, pp. 360-371, 2008.

[7] V. Babaei, S. H. Amirshahi and F. Agahian, " Using weighted pseudoinverse method for reconstruction of reflectance spectra and analyzing the dataset in terms of normality," Color Research \& Application, vol. 36, no. 4, pp. 295-305, 2011.

[8] W. F. Zhang and D. Q. Dai, "Spectral reflectance estimation from camera responses by support vector regression and a composite model," l. JOSA A, vol. 25, no. 9, pp. 2286-2296, 2008.

[9] F. H. Imai, "Spectral-based Imaging Techniqes and the Image Quality Challenge," in Ninth International Symposium on Multispectral Colour Science and Application, IS\&T, 2007.

[10] M. Yamaguchi, Y. Murakami, H. Hashizume, H. Haneishi, Y. Kanno and Y. Komiya, "High-fidelity color video reproduction of open surgery by six-band camera," Medical Imaging 2010:Image Perception, Observer Performance, and Technology Assessment, vol. 7627, p. 762707, 2010.

[11] ICC, "Proofing and printing documents that include spot inks," 2002 2018. [Online]. Available: http://www.color.org/CxF_test.xalter.

[12] K. Deshpande and P. Green, "A simplified method of predicting the colorimetry of spot color overprints," In Color and Imaging Conference, vol. 1, pp. 213-216, 2010.

[13] C. Lin, J. Xu and J. Xu, "Prediction Algorithm of Spectral Reflectance of Spot Color Ink Based on Color Parallel and Superposition Model," in Advanced Materials Research, 2012.

[14] S. Zuffi, R. Schettini and G. Mauri, "Spectral-based printer modeling and characterization," Journal of Electronic Imaging, vol. 14, no. 2, p. $023008,2005$.

[15] J. Guo, H. Xu, M. R. Luo and B. Wang, "Spectral characterisation of colour printer based on a novel grey component replacement method.," Chinese Optics Letters 9, no. 9, pp. 073301-073301, 2011.

[16] K. D. A. C. A. Wilkie and W. Purgathofer, "Tone reproduction and physically based spectral rendering," in Eurographics., 2002. 
[17] J. Meng, F. Simon, J. Hanika and C. Dachsbacher, "Physically meaningful rendering using tristimulus colors," vol. 34, no. 4, pp. 3140)., 2015.

[18] R. A. Hall and D. P. Greenberg, " A testbed for realistic image synthesis," IEEE Computer Graphics and Applications, vol. 3, no. 8, pp. 10-20, 1983.

[19] M. Yamaguchi, H. Haneishi, H. Fukuda, J. Kishimoto, H. Kanazawa, M. Tsuchida, R. Iwama and N. Ohyama, "High-fidelity video and stillimage communication based on spectral information: Natural vision system and its applications.," Spectral Imaging: Eighth International Symposium on Multispectral Color Science, vol. 6062, p. 60620G, 2006.

[20] J. R. Bintz, M. J. Mendenhall, M. A. Marciniak, S. D. Butler and J. T. Lloyd, "A novel image-based BRDF measurement system and its application to human skin," Reflection, Scattering, and Diffraction from Surfaces V, vol. 9961, p. 996108, 2016.

[21] A. S. Sole, I. Farup and T. S, "An image-based multi-directional reflectance measurement setup for flexible objects," Measuring, Modeling, and Reproducing Material Appearance. International Society for Optics and Photonics, vol. 9398, p. 93980J, 2015.

[22] A. Sole, I. Farup and S. Tominaga, "Image based reflectance measurement based on camera spectral sensitivities," in Electronic Imaging, 2016.

[23] S. R. Marschner, S. H. Westin, E. P. Lafortune and K. E. Torrance, "Image-based bidirectional reflectance distribution function measurement," Applied optics, vol. 39, no. 16, pp. 2592-2600, 2000.

[24] K. F. Karner, H. Mayer and M. Gervautz, "An image based measurement system for anisotropic reflection," Computer Graphics Forum, vol. 15, no. 3, pp. 119-128, 1996.

[25] A. Sole, I. Farup and P. B. Nussbaum, "Evaluating an image based multi-angle measurement setup using different reflection models," 2017.

[26] M. E. L. Leow, W. K. M. Ng, B. P. Pereira, A. K. Kour and R. W. H. Pro, "Metamerism in aesthetic prostheses under three standard illuminants-TL84, D65 and F," Prosthetics and orthotics international 23, no. 2, pp. 174-180, 1999.

[27] L. G. Coppel, S. Le Moan, P. Z. S. R. Elias and J. Y. Harderberg, "Next generation printing-Towards spectral proofing," Advanced in Printing and Media Technology, vol. 41, pp. 19-24, 2014.

[28] P. Urban and R. S. Berns, "Paramer mismatch-based spectral gamut mapping," IEEE transactions on image processing, vol. 20.6, pp. 1599-1610, 2010.

[29] M. D. Fairchild and R. L. Heckaman, "Measuring observer metamerism: The Nimeroff approach," Color Research \& Application, vol. 41, no. 2, pp. 115-24, 2015.

[30] A. Sarkar, "Identification and assignment of colorimetric observer categories and their applications in color and vision sciences," $\mathrm{PhD}$ diss, 2011.

[31] Y. Asano, "Individual colorimetric observers for personalized color imaging," 2015.
[32] B. Oicherman, M. R. Luo, B. Rigg and A. R. Robertson, "Effect of observer metamerism on colour matching of display and surface colours," Color Research \& Application: Endorsed by Inter-Society Color Council, The Colour Group (Great Britain), Canadian Society for Color, Color Science Association of Japan, Dutch Society for the Study of Color, The Swedish Colour Centre Foundation, Colour Soc, vol. 33, no. 5, pp. 346-359, 2008.

[33] D. Long and M. D. Fairchild, "Reducing observer metamerism in wide-gamut multiprimary displays," Human Vision and Electronic Imaging XX, vol. 9394, p. 93940T, 2015.

[34] I. C. Consortium, "Icc.2:2018 Image technology colour management extensions xtensions to architecture, profile format, and data structure," [Online]. Available: http://www.color.org/specification/ICC.2-2019.pdf.

[35] 2. International ColorConsortium, "iccMAX multiprocessingelement calculator programming," 2018. [Online]. Available: http://www.color.org/whitepapers/ICC White Paper45_Calculator Programming-v3.pdf.

[36] J. Vogh, "3D Appearance Management using iccMax," in ICC Meeting on Display and 3D Printing, Taiei, 2016, http://www.color.org/events/taipei/73D_Appearance_Management_using_iccMAX.pdf. 


\section{JOIN US AT THE NEXT EI!}

IS\&T International Symposium on

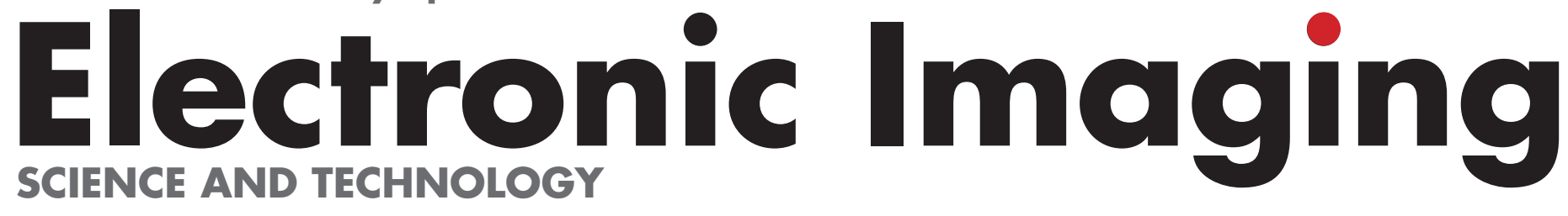

Imaging across applications ... Where industry and academia meet!
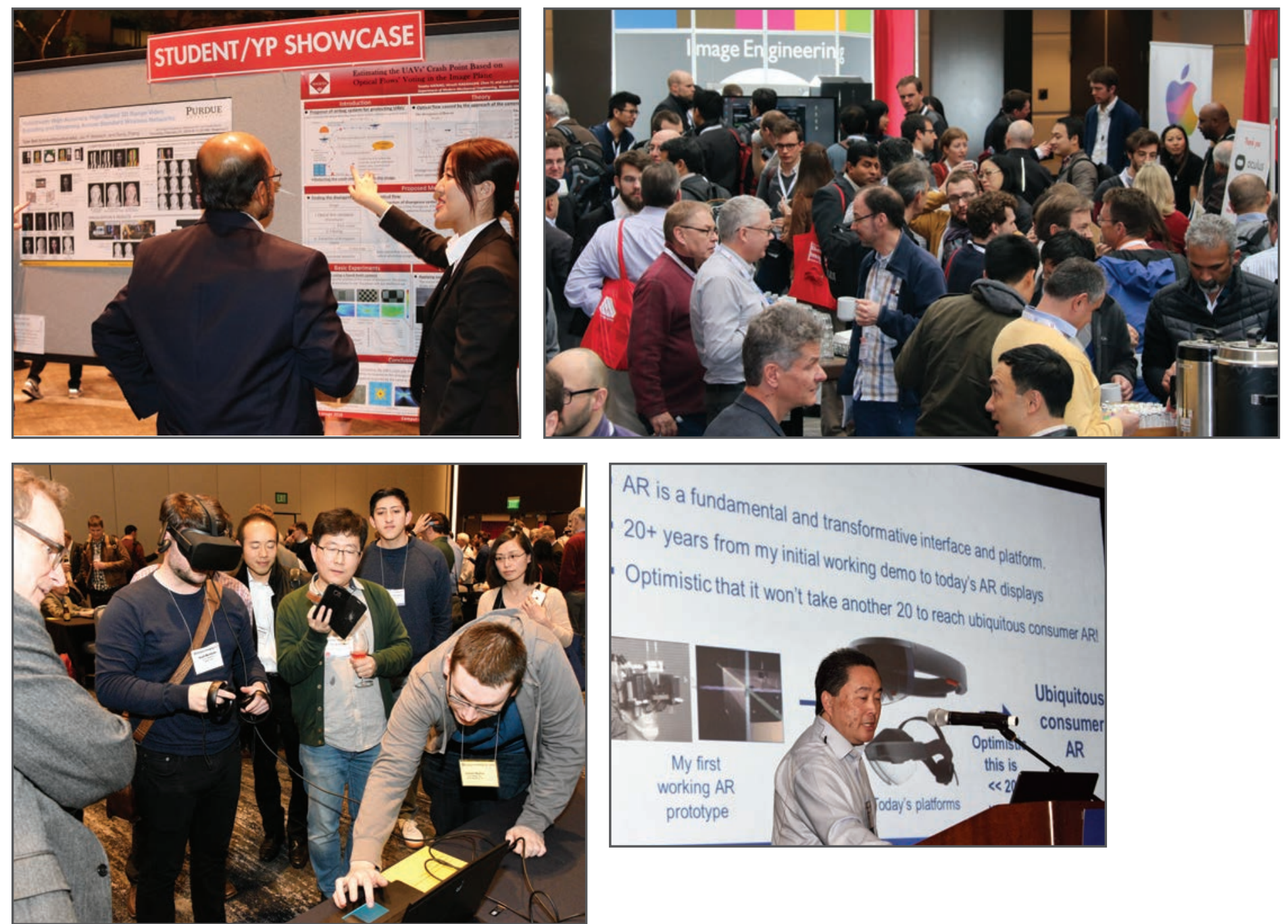

- SHORT COURSES • EXHIBITS • DEMONSTRATION SESSION • PLENARY TALKS •

- INTERACTIVE PAPER SESSION • SPECIAL EVENTS • TECHNICAL SESSIONS •

www.electronicimaging.org

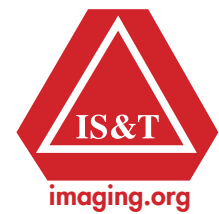

